\title{
Transcriptome Analysis Reveals Key Genes and Pathways Associated with Egg Production in Nandan-Yao Domestic Chicken
}

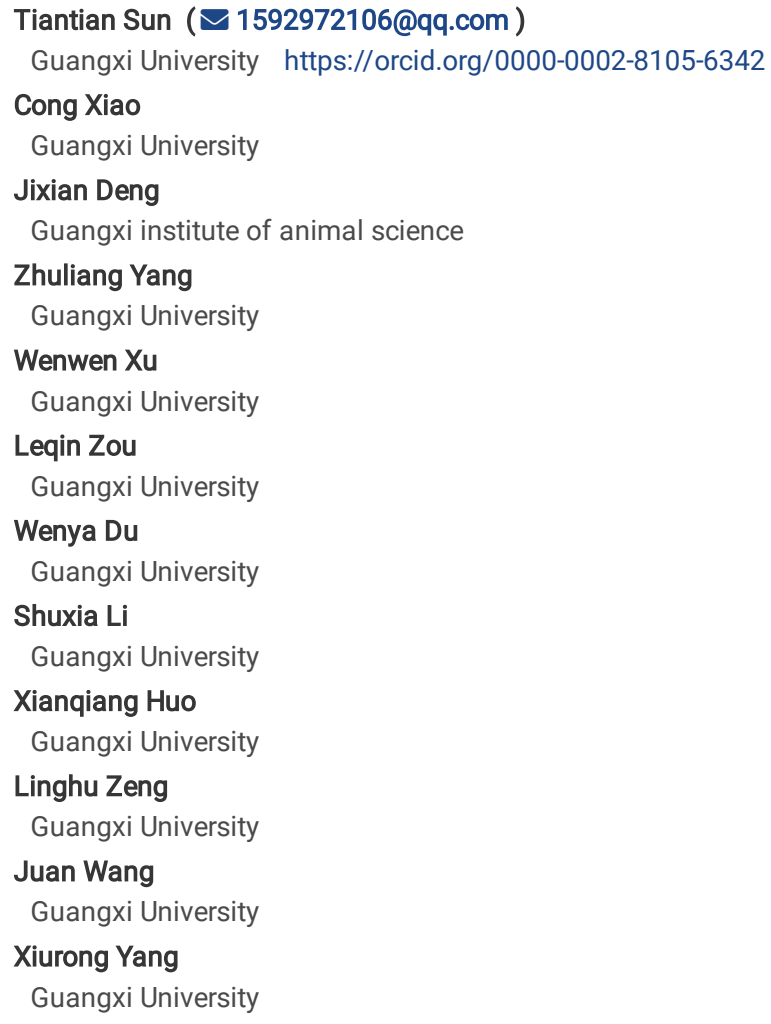

Version of Record: A version of this preprint was published at Comparative Biochemistry and Physiology Part D: Genomics and Proteomics on August 1st, 2021. See the published version at https://doi.org/10.1016/j.cbd.2021.100889. 


\section{Abstract \\ Background}

Egg production is a very important economic trait in chicken breeding, but its molecular mechanism is unclear until now. Nandan-Yao chicken (Gallus gallus domesticus) is a native breed in Guangxi province, China, which is famous for good meet quality, but low egg production. To explore the molecular regulation related egg production, high egg production (HEP) and low egg production (LEP) were divided according to the total egg number at 50 weeks, and the concentration of serum sex hormones was tested to evaluate the physiological function of ovary and uterus. RNA sequencing (RNA-Seq) was used to explore the transcriptome from the ovary and uterus of Nandan-Yao chicken.

\section{Results}

The levels of serum sex hormone were showed that concentrations of estradiol (E2), follicle-stimulating hormone (FSH), and luteotropic hormone (LH) were very significantly higher in HEP compared with LEP respectively $(P<0.01)$, and concentrations of testosterone (T) were very significantly lower in HEP compared with LEP $(P<0.01)$, which indicated there were better physiological function in HEP compared with LEP. Analysis results of RNA-Seq showed that 901 and 2763 differentially expressed genes (DEGs) in ovary and uterus between HEP and LEP chicken, respectively. Enrichment analysis of DEGs showed that DEGs were involved significantly in the regulation of tight junction in the ovary $(P<0.05)$, while in uterus DEGs were mainly enriched significantly in the phagosome, ECM-receptor interaction, cell adhesion molecules (CAMs), focal adhesion, cardiac muscle contraction, cytokine-cytokine receptor interaction, and the regulation of MAPK signaling pathway $(P<0.05)$. Protein network interaction and function analyses revealed FN1, FGF7, SOX2,ALDOB, HSPA2 in the ovary, and UQCRH, COX5A, FN1, TGFB, ACTN1 in the uterus were key candidate genes for egg production in Nandan-Yao chicken.

\section{Conclusions}

The current study identified key genes and pathway contribute to improving our understanding of reproductive biology of chicken and isolating effective molecular markers that can be used for genetic selection in Nandan-Yao chicken.

\section{Background}

Increasing egg production is one of the main purposes of breeding in laying hens[1]. The selection of egg-laying birds based on partial egg production can increase total egg production [2]. The interval of generations per time unit can be reduced by half if the selection is done considering partial egg production according to the previous study[3]. The selection of partial, or annual, egg counts or egg production rates is a common method for improving the laying performance of hens, which can produce some positive genetic progress but it is slow[4]. However, most of the chicken laying traits, including egg production, egg weight, and age of sexual maturity, are polygenic traits with low to moderate heritability, making it difficult to estimate the level of genetic improvement in each generation[5].

The quest for improved egg production is an important focus of poultry breeding. A large number of people have done a lot of research on egg production through different technologies. For example, the genome-wide association study identified many mutants associated with egg production by chickens[6]. Wang CQ et al (2019) using RNA-seq for high yield and low yield Dagu China chicken (CDC) of the hypothalamus and pituitary expression profiles for sequencing and analysis of the transcriptome, functional annotation and pathway enrichment analysis showed that DEGs mainly rich in sugar glycosaminoglycans biosynthesis, extracellular matrix, protein extracellular matrix and extracellular space[1]. N.Wu et al performed the miRNA analysis of ovarian tissues in chickens with low and high rates of egg production using high-throughput sequencing. They found some significantly differentially expressed ovarian miRNAs, such as gga-miR-1744-3p, ggamiR-1655-5p, gga-miR-1734, and gga-miR-7465-3p, in the high egg-laying chickens[7]. Single nucleotide polymorphism (SNP) is a polymorphism of the DNA sequence produced by a single base mutation (base replacement, insertion or deletion, etc.), which is the most common form of variation in the genome[8]. Bhattacharya T K et al. (2019) found that the polymorphism of GnRHIand GnRHII genes was significantly correlated with egg production and body weight of white leghorn laying hens[9]. Li G et al. (2011) analyzed the effect of FSHR gene polymorphism on egg production in the Beijing oil chicken population. The correlation analysis showed that the polymorphic loci of $\mathrm{g} 310 \mathrm{~A}>\mathrm{g}$, $\mathrm{g} 181 \mathrm{~A}>\mathrm{T}$, and g159 C > T were all correlated with the number of eggs in Beijing-You chicken population of different weeks $(P<0.05)[10]$. Han HX et al. (2015) studied the relationship between BMP15 gene polymorphism and reproductive traits of LaiWu Black chicken and identified three single nucleotide polymorphisms (SNPs) [11]. Sex hormone regulation also affects the development of sex organs and egg production. Estrogen promotes the maturation of animal reproductive organs. Estrogen cooperates with FSH to promote follicular development, inducing the emergence of LH secretion peak before ovulation and promoting ovulation. Egg-laying traits are complex traits controlled by multiple genes. However, at present, the number of genes associated with egg production remains very limited, and little is known about the links among these genes.

RNA-seq is a methodology for RNA profiling based on next-generation sequencing that enables us to measure and compare gene expression patterns at an unprecedented resolution[12]. In recent years, to further explore the molecular mechanisms that regulate important economic traits of poultry, a large number of poultry transcriptome studies have been performed using RNA-Seq[7, 13-16]. The development of RNA sequencing has provided a powerful, highly reproducible, and cost-efficient tool for exploring the molecular mechanism of traits.

The Nandan-Yao chicken is a native chicken breed in China. It has the characteristics of coarse food resistance, strong foraging ability, delicate and delicious meat, but its performance of egg production is low. There are large differences among individuals in Nandan-Yao chicken population, which provides good material for the study of the mechanism of egg production. High egg production has an important economic value in chicken breeding, the current study aims 
to find some key genes and signaling pathways influencing egg production of Nandan-Yao chicken. In the present study, we sampled the ovarian and uterine tissues of three LEP and three HEP Nandan-Yao chickens for RNA-sEq. These data will contribute to the investigation of the regulatory mechanism of egg production.

\section{Results}

\section{The concentration of serum sex hormone}

Concentrations of E2, T, FSH, LH in Nandan-Yao chicken serum are shown below(Fig. 1). The results showed that the concentration of E2 in the HEP was significantly higher than that in the LEP $(P<0.01)$, the concentration of T in the HEP was significantly lower than that in the LEP $(P<0.01)$, and the E2/T in the HEP was significantly higher than that in the LEP $(P<0.01)$ (Fig. 1A). The concentration of FSH and LH in the HEP was significantly higher than that in the LEP $(P<0.01)$ (Fig. 1B). These results indicated there was a better physiological function in HEP compared with LEP.

\section{Transcriptome data}

The results of transcriptome sequencing and mapping information are shown in Table 1. Total sequencing data for each sample were $77,056,834$ to $105,340,082$. The average GC content was $47.90 \%$, the percentage of Q20 base was more than $95.94 \%$, the average mapping was $91.24 \%$.

Table 1

Summary of sequencing reads mapping to the reference genome and quality parameters

\begin{tabular}{|llllll|}
\hline Sample name & Raw reads & Clean reads & Q20(\%) & Total mapped (\%) & GC content(\%) \\
\hline H1-ovary & 77056834 & 76852266 & 95.94 & 90.05 & 48.75 \\
\hline H2-ovary & 96943330 & 96520578 & 97.19 & 91.25 & 46.94 \\
\hline L1-ovary & 100526350 & 100231364 & 96.96 & 91.8 & 47.11 \\
\hline L2-ovary & 93188406 & 92488460 & 97.21 & 90.44 & 48.68 \\
\hline L3-ovary & 89862614 & 89604478 & 96.68 & 90.98 & 48.22 \\
\hline H1-uterus & 89109406 & 88774844 & 97.34 & 91.84 & 49.04 \\
\hline H2-uterus & 88484558 & 88156120 & 97.25 & 91.52 & 46.77 \\
\hline H3-uterus & 91664794 & 91319256 & 97.48 & 91.83 & 45.77 \\
\hline L1-uterus & 102667456 & 102255290 & 97.33 & 91.31 & 47.1 \\
\hline L2-uterus & 105340082 & 104907586 & 97.17 & 90.26 & 49.12 \\
\hline L3-uterus & 89507722 & 89248116 & 96.96 & 90.91 & 50.09 \\
\hline
\end{tabular}

\begin{tabular}{|c|c|c|c|c|}
\hline Tissue & Pathways & $\begin{array}{l}\text { DEGs } \\
\text { No. }\end{array}$ & P-value & Genes \\
\hline ovary & Tight junction & 20 & 0.007772 & RAPGEF6,CFTR,MYL12B,AMOTL1,PRKACB,MAP3K5,TUBA3E,PRKAG2,NEDD4,TJP1,BVES,ACTB, MYH10, \\
\hline \multirow[t]{8}{*}{ uterus } & $\begin{array}{l}\text { Oxidative } \\
\text { phosphorylation }\end{array}$ & 38 & 0.000069 & ATP5L,SDHB,ATP5G2,COX7A2,COX7B,UQCRHL,UQCR11,NDUFB4,COX5A,ATP5I,COX7C, ATP6AP1 UQCRC \\
\hline & Phagosome & 38 & 0.002209 & BLB2, CYBA, TUBA4A, MMR1L4, BF1, BLB1, ATP6AP1, TAP1, NOS1, CTSS,BF2,ATP6V1G3,DYNC1I2,PIKFI \\
\hline & $\begin{array}{l}\text { ECM-receptor } \\
\text { interaction }\end{array}$ & 27 & 0.002604 & ITGA7,COL9A1,CHAD,SV2C,LAMA3,LAMB3,NPNT,LAMA4,VWF,ITGB4,ITGA11,THBS2,COL6A3,LAMB1,TNC \\
\hline & $\begin{array}{l}\text { Cell adhesion } \\
\text { molecules } \\
\text { (CAMs) }\end{array}$ & 33 & 0.010032 & CD6 BLB2,C6H10orf54, CD80,BF1,LRRC4C, NCAM1,CLDN8,PDCD1LG2,BF2,PDCD1, PTPRF, CTLA4, ICOS,I \\
\hline & Focal adhesion & 46 & 0.010032 & ITGA7, PPP1CA,COL9A1, PDGFRB, MYL12B, CHAD,LAMA3,JUN,VEGFC,LAMA4,VWF PARVG, RAC2,ITGB4, \\
\hline & $\begin{array}{l}\text { Cardiac muscle } \\
\text { contraction }\end{array}$ & 21 & 0.010032 & 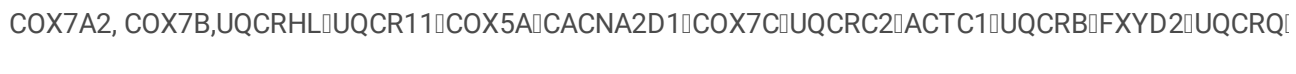 \\
\hline & $\begin{array}{l}\text { Cytokine- } \\
\text { cytokine } \\
\text { receptor } \\
\text { interaction }\end{array}$ & 48 & 0.010186 & CSF2RB,IL18RAP $I L 2 R B \square C S F 1 \square C C R 6 \square C X C R 1 \square C X C R 7 \square X C R 1 \square C C L 19 \square I L 7 R \square N G F R \square I L 12 R B 2 \square A M H R 2 \square B M P 3 \square I$ \\
\hline & $\begin{array}{l}\text { MAPK signaling } \\
\text { pathway }\end{array}$ & 56 & 0.048725 & 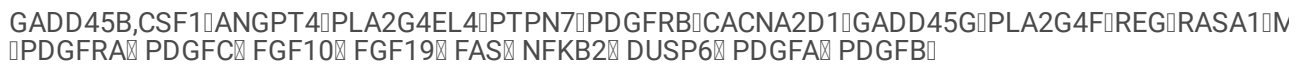 \\
\hline
\end{tabular}




\section{Analysis of Differentially Expressed Genes}

In the ovary, 901 genes were identified to be significantly DEGs, among of them, 472 and 429 genes were down-regulated and up-regulated, respectively (Fig. 2A). In the uterus, we identified 2763 DEGs, including 1786 down-regulated genes and 977 up-regulated genes, respectively (Fig. 2B). The 299 DEGs displayed differential expression both in ovary and uterus(Fig. 2C). Hierarchical cluster analysis was used for viewing similarities between different samples. Figure 3 shows that individuals in HEP and LEP were all clustered together which illustrates the accuracy of the sample and the reliability of the differentially expressed genes obtained by our bioinformatics analysis.

\section{GO and KEGG analysis for DEGs}

Gene Ontology(GO)enrichment and Kyoto Encyclopedia of Genes and Genomes $\square$ KEGG $\square$ analysis was used to evaluate gene ontology and signaling pathway of DEGs(Fig. 4B). GO enrichment of DEGs in ovary showed no significant enrichment terms $(P<0.05)$. GO enrichment of DEGs in uterus revealed that there were 82 significantly enriched terms in the three categories $(P<0.05)$. The top 10 significantly enriched $\mathrm{GO}$ terms were shown in Fig. $4 \mathrm{~A}$, including extracellular structure organization, extracellular matrix organization, anatomical structure morphogenesis, ion homeostasis, cation homeostasis, inorganic ion homeostasis, blood vessel development, chemical homeostasis, regulation of the multicellular organismal process, cellular ion homeostasis. KEGG enrichment of DEGs in ovary showed that there was one significant enrichment pathway(tight junction). KEGG enrichment of DEGs in the uterus was significantly enriched in the 8 signaling pathways of oxidative phosphorylation, phagosome, ECM-receptor interaction, cell adhesion molecules (CAMs), focal adhesion, cardiac muscle contraction, cytokine-cytokine receptor interaction, MAPK signaling pathway $(P<0.05)$.

\section{Integration of PPI network and module analysis}

The DEG network interaction analysis of ovary and uterus is shown in Fig. 5. Ovarian tissue contains 77 genes and 175 interaction relationships, uterine tissue contains 428 genes and 2962 interaction relationships. The top five genes in the ovary included ACTB, GAPDH, FN1, FGF7, and SOX2; the top five genes in the uterus included UQCRH, COX5A, ACTB, FN1, and GAPDH. These genes may play an important regulatory role in the laying process. Due to a large number of DEGs in uterine, we built the top three important modules in the PPI network of uterine DEGs through the MCODE application(Fig. 6). Module 1 (MCODE score $=18.579)$ included 37 genes, 331 interactions. Module $2($ MCODE score $=10)$ included 38 genes and 185 interactions. Module $3($ MCODE score $=6)$ includes 6 genes and a total of 15 interactions.

\section{Validation of RNA-seq}

To verify our RNA-seq data, we selected 13 DEGs related to gonad development (AMH, SOX9, FOXL2, CYP19A1, ESR1, ESR2, BMPER, GREM1, FSHR), yolk production(VTG2, PPAR-y) and antioxidant (GLRX, TXN) for QRT-PCR analysis (Fig. 7), which were common DEGs in ovary and uterus of Nandan-Yao chicken. The results showed that the differentially expressed genes had the same expression trends in QRT-PCR and RNA-seq, which validated their accuracy.

\section{Discussion}

Although many studies have been conducted on egg production, the molecular mechanisms are still unclear. In this study, we employed RNA-seq to sequenced transcriptomes of two tissues (ovary and uterus) in three high- and three low-egg production individuals.

In this study, we identified 901 and 2763 DEGs in ovary and uterus, respectively. Of these, 118 and 140 DEGs were up-regulated and down-regulated in the ovary by $\log _{2} \mid$ foldchange|> 4 while 156 and 298 DEGs were up-regulated and down-regulated in the uterus. These results suggest that these DEGs may play important roles in the regulation of egg production. For example, SPP1 was a candidate gene related to the formation of the egg and oviposition[17, 18]. Studies identified a candidate gene BPIFB3 closely located on chromosome 20 is involved in the development of the reproductive system based on the genome-wide association study results[19]. The Glycoproteins EDIL3 regulates vesicle-mediated eggshell calcification in avian biomineralization[20].

The DEGs detected in the ovary were mainly enriched in the tight junction $(P<0.05)$, while the DEGs detected in the uterus were mainly enriched in the phagosome, ECM-receptor interaction, cell adhesion molecules (CAMs), focal adhesion, cardiac muscle contraction, cytokine-cytokine receptor interaction, and the regulation of MAPK signaling pathway $(P<0.05)$. Tight junctions in the mucosal epithelium have essential roles as a mucosal barrier to prevent the invasion of microbes into the hen oviduct tissue[21]. Studies have shown that the level of oxidative phosphorylation strongly influences oocyte quality[22]. ECM-receptor interaction, focal adhesion, and cytokine-cytokine receptor interaction pathways were enriched in other studies[23, 24].

Protein network interaction and function analyses revealed FN1, FGF7, SOX2,ALDOB, HSPA2 in the ovary, and UQCRH, COX5A, FN1, TGFB, ACTN1 in the uterus were key candidate genes for egg production in Nandan-Yao chicken. Studies have shown that FN1 may be involved in the regulation of the MII stage oocyte formation and several other processes that are crucial for porcine reproductive competence[25]. Studies have shown that FGF7 may be an important regulator for oocyte growth and its action is mediated via the KIT/KITLG signaling pathway[26]. Sox2 was a critical transcriptional regulator in the oocyte-to-embryo transition[27]. The loss of COX5A leads to mitochondrial dysfunction that results in apoptosis of the blastocyst stage embryos[28]. Studies have shown that the distribution pattern of TGFB superfamily genes is associated with the stage of maturation of porcine oocytes and the follicle size[29]. The analysis of the PPI network showed that ATP6V0A1, ATP6V1C2, ATP6AP1, ATP6V0D2, ATP6V1G3, and ATP6V1A genes interacted (Fig. 6C). At the same time, the results of KEGG analysis showed the ATP6V0A1, ATP6V1C2, ATP6AP1, ATP6V0D2, ATP6V1G3, and ATP6V1A gene was significantly enriched ( $P<0.05)$ to 2 signaling pathways in the uterus, Oxidative phosphorylation, and Phagosome. The results of G0 analysis showed the ATP6V0A1, ATP6V0D2, and ATP6V1A gene were significantly enriched $(P<0.05)$ to 7 molecular functions related to transmembrane movement of substances in the uterus. These molecular functions include cation-transporting ATPase activity, active ion transmembrane transporter activity, ATPase coupled ion transmembrane transporter activity, primary active 
transmembrane transporter activity, P-P-bond-hydrolysis-driven transmembrane transporter activity, ATPase activity coupled to transmembrane movement of substances, ATPase activity coupled to the movement of substances. It is possible that a large amount of energy generation and the transmembrane movement of substances is caused by egg production.

\section{Conclusions}

The current study identified a series of key genes and signaling pathways associated with egg production by RNA-seq and bioinformatics analysis. These key genes may regulate egg production by taking part in tight junction, phagosome, ECM-receptor interaction, cell adhesion molecules (CAMs), focal adhesion, cardiac muscle contraction, cytokine-cytokine receptor interaction, and the regulation of MAPK signaling pathway. The current findings provide very important information for understanding the molecular mechanism of egg production and developing effective molecular markers for the molecular breed in NandanYao domestic chicken.

\section{Methods}

\section{Ethics statement}

All experimental and sample collection procedures were approved by the Institutional Animal Care and Use Committee (IACUC) of the College of Animal Science and Technology of Guangxi University (Guangxi, China), with approval number GXU2018-058.

\section{Animals and Sample Collection}

A total of 300 Nandan-Yao chickens were bred in poultry experimental farm of Guangxi University, which were purchased from Guangxi Guigang Gangfeng Agriculture and Husbandry Co. Ltd. All layers were housed in individual pen of the same feeding and management condition. At the end of 50wks, the chickens were divided into high egg production (HEP) and low egg production (LEP) according to the total egg number. We selected three chickens from each HEP and LEP group as the fnal experimental birds(Table S1). The average egg number was $82 \pm 1.4$ and $185 \pm 7.8$ (Mean \pm S.E.M.) for LEP and HEP chickens, respectively. Ovary and uterus were collected, then quickly frozen in liquid nitrogen and transfer into $-80^{\circ} \mathrm{C}$ for permanent preservation until RNA extraction.Blood samples for testing the concentration of serum sex hormone were collected and the serum was isolated from blood by centrifuging.

\section{Test of serum hormone concentrations}

A serum sex hormone was tested by using Chicken E2, T, FSH, LH ELISA Kit (Shanghai Enzyme Biotechnology Co., Ltd.). The concentration of the sex hormone was calculated according to the standard curve. The t-test was used to test the significant differences between LEP and HEP.

\section{Total RNA extraction}

The total RNA was extracted from ovary and uterus using Trizol reagent (Life Technologies, USA) according to the manufacturer's instruction and modified a little. The quality and quantity of total RNA were tested according to the results of agarose gel electrophoresis and testing of UV-Vis Spectrophotometer Q5000 (Quawell, USA).

\section{RNA sequencing and quality control}

The cDNA libraries were constructed and sequenced on an Illumina HiSeq 2500 (Illumina, San Diego, CA, USA) in Novogene Bioinformatics Technology Co., Ltd., Beijing, China. RNA-seq was conducted following the manufacturer's standard procedures. Raw data were first processed through trim galore [30], and clean data were obtained by removing reads containing adapter sequences, reads containing poly- $\mathrm{N}$, and low-quality reads from the raw data. Furthermore, use FastQC for quality control of filtered data quality parameters of Q20, GC content, and sequence duplication levels were used for data filtering[31].

\section{RNA-Seq analysis}

Reference genome and gene model annotation files were downloaded from the genome website (ftp://ftp.ensembl.org/pub/release-

95/fasta/gallus_gallus/dna/). Paired-end clean reads were aligned to the reference genome using Hisat2v2.1.0[32, 33]. Hisat2 was selected as the mapping tool because it can be faster and more precise than other splice mapping tools as well as obtain soft-splices information than other no splice mapping tools[34]. The stringtiev2.1.1 reference annotation-based transcript assembly method was used to construct and identify both known and novel transcripts from Hisat2 alignment results[35]. The differentially expressed genes analysis was performed using the DESeq2 R package (1.18.0)[36] and the criteria for screening differentially expressed genes were $\mathrm{p}$-value $<0.05$.

\section{Bioinformatics analysis}

All DEGs were subjected to GO and KEGG pathway enrichment analysis by the R package clusterProfiler 3.14.3[37]. The parameters were set as minGSize $=3$, p-value cutoff $=0.2$, and q-value cutoff $=0.2$,ont $=$ all. The STRING[38] database is used to explore the interaction between DEGs. A confidence score $>0.9$ was defined as valid. Submit STRING analysis results to Cytoscape[39] (http://cytoscape.org/) for visualization, and use the CytoHubba application in Cytoscape to find key hub genes through the MCC algorithm[40]. Module detection (MCODE) in Cytoscape[41] was used to screen key modules in the PPI network. The standard setting of MCODE was degree cutoff $=2$, node score cutoff $=0.2, \mathrm{k}$-core $=2$, maximum depth $=100$.

\section{Validation of RNA-Seq}

13 genes were selected from DEGs randomly for accuracy testing of RNA-SEq. Total RNA was isolated by Trizol as above from ovary and uterus and the first chain of cDNA was synthesized by reverse transcription kit (Takara, Dalian, China). The Quantitative Real-time PCR(QRT-PCR) was performed according to the following program: the volume of the reaction mixture was $20 \mu \mathrm{l}$, with $2 \mu \mathrm{l}$ of cDNA, $0.5 \mu \mathrm{l}$ of primers, $10 \mu \mathrm{l}$ of SYBR (Takara, Dalian, China), and $7 \mu \mathrm{l}$ of RNA- 
free water. Then the QRT-PCR was run at $95^{\circ} \mathrm{C}$ for $3 \mathrm{~min}$; followed by 35 cycles of $95^{\circ} \mathrm{C}$ for $10 \mathrm{~s}$. $\beta$-Actin was taken as a house keep gene to correct the gene expression levels. The $2^{-\triangle \Delta C T}$ method was used to calculate relative expression levels. The t-test was used to test the significant differences among genes.

\section{Abbreviations}

HEP: high egg production; LEP: low egg production; RNA-Seq: RNA sequencing; E2: estradiol; FSH: follicle-stimulating hormone; LH: luteotropic hormone; T: testosterone ; DEGs: differentially expressed genes ; CAMs: cell adhesion molecules; SNP: Single nucleotide polymorphism; CDC: Dagu China chicken ;GO: Gene Ontology ;KEGG: Kyoto Encyclopedia of Genes and Genomes;QRT-PCR: Quantitative Real-time PCR.

\section{Declarations}

\section{Ethics approval and consent to participate}

All experimental and sample collection procedures were approved by the Institutional Animal Care and Use Committee (IACUC) of the College of Animal Science and Technology of Guangxi University (Guangxi, China), with approval number GXU2018-058.

\section{Consent for publication}

Not Applicable.

\section{Availability of data and materials}

The datasets used and/or analyzed during the current study are available from the corresponding author on reasonable request.

\section{Competing interests}

The authors declare that they have no competing interests.

\section{Funding}

This work was supported by grants from Guangxi innovation-driven development special fund project (Guike AA17204027); Science and technology project of Guigang science and technology bureau (1829010). The funding body had no role in the design of the study, the collection,analysis, and interpretation of data, and in writing the manuscript and the decision to submit the work for publication.

\section{Authors' contributions}

$\mathrm{XY}$ and TS designed the study. TS,CX,JD,ZY,LZ,WD,SL,XH,LZ,JW,XY performed the experiment, sample preparation and bioinformatic analyses. TS and $X Y$ wrote the manuscript. All authors approved the final manuscript.

\section{Acknowledgements}

Not Applicable

\section{References}

1. Wang C, Ma W. Hypothalamic and pituitary transcriptome profiling using RNA-sequencing in high-yielding and low-yielding laying hens. Sci Rep. 2019;1(9):10285.

2. Poggenpoel DG, Ferreira GF, Hayes JP, du Preez JJ. Response to long-term selection for egg production in laying hens. British poultry science. 1996;37(4):743-56.

3. Silva MA, Euclydes RF, Soares PR. and J. B. Fonseca.: Análise genética de características de importância econômica em poedeiras leves. $R$ Bras Zootec 1984(13):82-94.

4. Fairfull RW, Gowe RS. Genetics of egg production in chickens.. Poultry Breeding and Genetics 1990.

5. Biscarini F, Bovenhuis H, Ellen ED, Addo S, van Arendonk JA. Estimation of heritability and breeding values for early egg production in laying hens from pooled data. Poultry science. 2010;89(9):1842-9.

6. Yuan J, Sun C, Dou T, Yi G, Qu L, Qu L, Wang K, Yang N. Identification of Promising Mutants Associated with Egg Production Traits Revealed by GenomeWide Association Study. PLoS One. 2015;10(10):e0140615.

7. Wu NG, Zhu U, Chen Q, Xu B, Zhao Z, Yang X, Li M. D.: Expressed microRNA associated with high rate of egg production in chicken ovarian follicles. Anim Genet. 2017;48:205-16.

8. Yan ZG, Peterson AH, Zhong ZP, Bendixen C. The methods and principles for scanning and genotyping single nucleotide polymorphisms (SNP). Animal Biotechnology Bulletin. 2000;2000:13-9. 
9. Bhattacharya TK, Chatterjee RN, Dange M, Bhanja SK. Polymorphisms in GnRHI and GnRHII genes and their association with egg production and egg quality traits in chicken. Br Poult Sci. 2019;3(60):187-94.

10. Li G, Sun DX, Yu Y, Liu WJ, Tang SQ, Zhang Y, Wang YC, Zhang SL, Zhang Y. Genetic effect of the follicle-stimulating hormone receptor gene on reproductive traits in Beijing You chickens. Poultry science. 2011;90(11):2487-92.

11. Han H, Lei Q, Zhou Y, Gao J, Liu W, Li F, Zhang Q, Lu Y, Cao D. Association between BMP15 Gene Polymorphism and Reproduction Traits and Its Tissues Expression Characteristics in Chicken PLoS One 2015, 11(10):e0143298.

12. Finotello F, Di Camillo B. Measuring differential gene expression with RNA-seq: challenges and strategies for data analysis. Brief Funct Genomics. 2015;14(2):130-42.

13. Yin Z, Lian L, Zhu F, Zhang ZH, Hincke M, Yang N, Hou ZC. The transcriptome landscapes of ovary and three oviduct segments during chicken (Gallus gallus) egg formation. Genomics. 2020;112(1):243-51.

14. Kang L, Cui X, Zhang Y, Yang C, Jiang Y. Identification of miRNAs associated with sexual maturity in chicken ovary by Illumina small RNA deep sequencing. BMC Genom. 2013;14:352.

15. Luan X, Liu D, Cao Z, Luo L, Liu M, Gao M, Zhang X. Transcriptome profiling identifies differentially expressed genes in Huoyan goose ovaries between the laying period and ceased period. PLoS One. 2014;9:e113211.

16. Adetula AA, Gu L, Nwafor CC, Du X, Zhao S, Li S. Transcriptome sequencing reveals key potential long non-coding RNAs related to duration of fertility trait in the uterovaginal junction of egg-laying hens. Scientific reports. 2018;8(1):13185.

17. Jeong W, Lim W, Kim J, Ahn SE, Lee HC, Jeong JW, Han JY, Song G, Bazer FW. Cell-specific and temporal aspects of gene expression in the chicken oviduct at different stages of the laying cycle. Biol Reprod. 2012;6(86):172.

18. Yang J, Li XY, Cao YH, Pokharel K, Hu XJ, Chen ZH, Xu SS, Peippo J, Honkatukia M, Kantanen J, et al. Comparative mRNA and miRNA expression in European mouflon (Ovis musimon) and sheep (Ovis aries) provides novel insights into the genetic mechanisms for female reproductive success. Heredity. 2019;2(122):172-86.

19. Dong X, Li J, Zhang Y, Han D, Hua G, Wang J, Deng X, Wu C. Genomic Analysis Reveals Pleiotropic Alleles at EDN3 and BMP7 Involved in Chicken Comb Color and Egg Production. Frontiers in genetics 2019(10):612.

20. Stapane L, Le Roy N, Hincke MT, Gautron J. The glycoproteins EDIL3 and MFGE8 regulate vesicle-mediated eggshell calcification in a new model for avian biomineralization. J Biol Chem. 2019;294(40):14526-45.

21. Ariyadi B, Isobe N, Yoshimura Y. Expression of tight junction molecule "claudins" in the lower oviductal segments and their changes with egg-laying phase and gonadal steroid stimulation in hens. Theriogenology. 2013;2(79):211-8.

22. Wilding M, Di Matteo L, Dale B. The maternal age effect: a hypothesis based on oxidative phosphorylation. Zygote. 2005;13(4):317-23.

23. Zhang T, Chen L, Han K, Zhang X, Zhang G, Dai G, Wang J, Xie K. Transcriptome analysis of ovary in relatively greater and lesser egg producing Jinghai Yellow Chicken. Anim Reprod Sci. 2019;208:106114.

24. Wu Y, Zhao X, Chen L, Wang J, Duan Y, Li H, Lu L. Transcriptomic Analyses of the Hypothalamic-Pituitary-Gonadal Axis Identify Candidate Genes Related to Egg Production in Xinjiang Yili Geese. Animals (Basel). 2020;10(1):90.

25. Budna J, Chachuła A, Kaźmierczak D, Rybska M, Ciesiółka S, Bryja A, Kranc W, Borys S, Żok A, Bukowska D, et al. Morphogenesis-related gene-expression profile in porcine oocytes before and after in vitro maturation. Zygote. 2017;3(25):331-40.

26. Cho JH, Itoh T, Sendai Y, Hoshi H. Fibroblast growth factor 7 stimulates in vitro growth of oocytes originating from bovine early antral follicles. Mol Reprod Dev. 2008;75(12):1736-43.

27. Pan H, Schultz RM. Sox2 Modulates Reprogramming of Gene Expression in Two-Cell Mouse Embryos. Biol Reprod. 2011;2(85):409-16.

28. Cui XS, Li XY, Jeong YJ, Jun JH, Kim NH. Gene Expression of cox5a, 5b, or 6 b1 and Their Roles in Preimplantation Mouse Embryos. Biol Reprod. 2006;3(74):601-10.

29. Jackowska M, Kempisty B, Woźna M, Piotrowska H, Antosik P, Zawierucha P, Bukowska D, Nowicki M, Jaśkowski J, Brüssow M. KP: Differential Expression of GDF9, TGFB1, TGFB2 and TGFB3 in Porcine Oocytes Isolated From Follicles of Different Size Before and After Culture in Vitro. Acta veterinaria Hungarica. 2013;1(61):99-115.

30. Bolger AM, Lohse M, Usadel B. Trimmomatic: a flexible trimmer for Illumina sequence data. Bioinformatics. 2014;30(15):2114-20.

31. Schmieder R, Edwards R. Quality control and preprocessing of metagenomic datasets. Bioinformatics. 2011;27(6):863-4.

32. Pertea M, Kim D, Pertea GM, Leek JT, Salzberg SL. Transcript-level expression analysis of RNA-seq experiments with HISAT, StringTie and Ballgown. Nature protocols. 2016;11(9):1650-67.

33. Kim D, Langmead B, Salzberg SL. HISAT: a fast spliced aligner with low memory requirements. Nature methods. 2015;12(4):357-60.

34. Sahraeian S, Mohiyuddin M, Sebra R, Tilgner H, Afshar PT, Au KF, Bani Asadi N, Gerstein MB, Wong WH, Snyder MP, et al. : Gaining comprehensive biological insight into the transcriptome by performing a broad-spectrum RNA-seq analysis. Nature communications. 2017;1(8):59.

35. Pertea M, Pertea GM, Antonescu CM, Chang TC, Mendell JT, Salzberg SL. StringTie enables improved reconstruction of a transcriptome from RNA-seq reads. Nature biotechnology. 2015;33(3):290-5.

36. Love MI, Huber W, Anders S: Moderated estimation of fold change and dispersion for RNA-seq data with DESeq2. Genome biology 2014, 15(12):550.

37. Yu G, Wang LG, Han Y, He QY. clusterProfiler: an R package for comparing biological themes among gene clusters. OMICS. 2012;5(16):284-7.

38. Franceschini A, Szklarczyk D, Frankild S, Kuhn M, Simonovic M, Roth A, Lin J, Minguez P, Bork P, von Mering C, et al. STRINGv9.1: protein-protein interaction networks, with increased coverage andintegration. Nucleic Acids Res. 2013;41(D1):D808-15.

Page $7 / 11$ 
39. Shannon PM, Ozier A, Baliga O, Wang NS, Ramage JT, Amin D, Schwikowski N, Ideker B. T.: Cytoscape: a software

Environmentforintegratedmodelsofbiomolecularinteraction networks. Genome Research 2003(13):2498-2504.

40. Liu Z, Meng J, Li X, Zhu F, Liu T, Wu G, Zhang L: Identification of Hub Genes and Key Pathways Associated with Two Subtypes of Diffuse Large B-Cell Lymphoma Based on Gene Expression Profiling via Integrated Bioinformatics. BioMed research international 2018, 2018:3574534.

41. Bader GD, Hogue CW. An automated method for finding molecular complexes in large protein interaction networks. BMC Bioinformatics. $2003 ; 4(4): 2$.

\section{Figures}
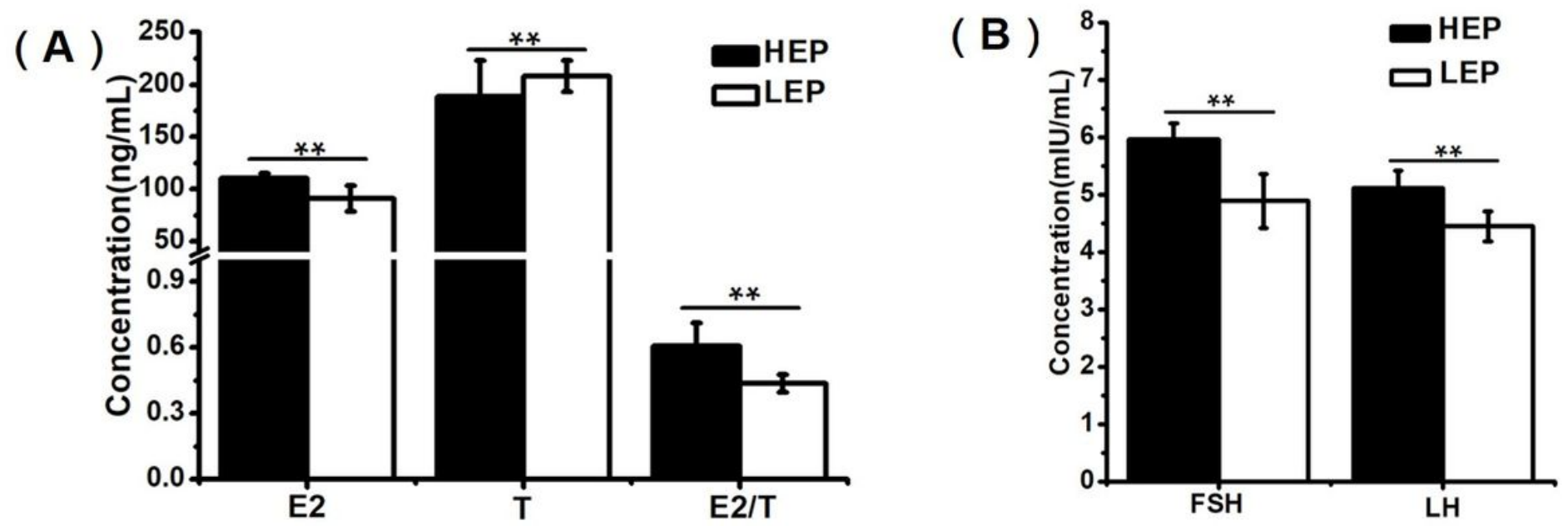

Figure 1

Serum sex hormone concentration in HEP and LEP. (A) the concentration of estradiol(E2) and testosterone (T), the ratio of E2/T in serum between HEP and LEP; (B) concentration of FSH and LH in serum between HEP and LEP. **present very significantly different, $\mathrm{P}<0.01$.

(A)

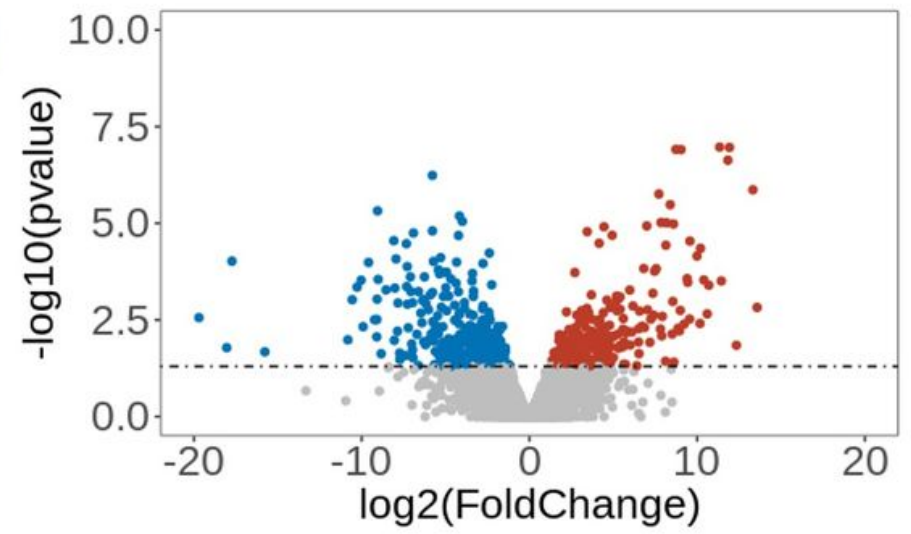

(B)

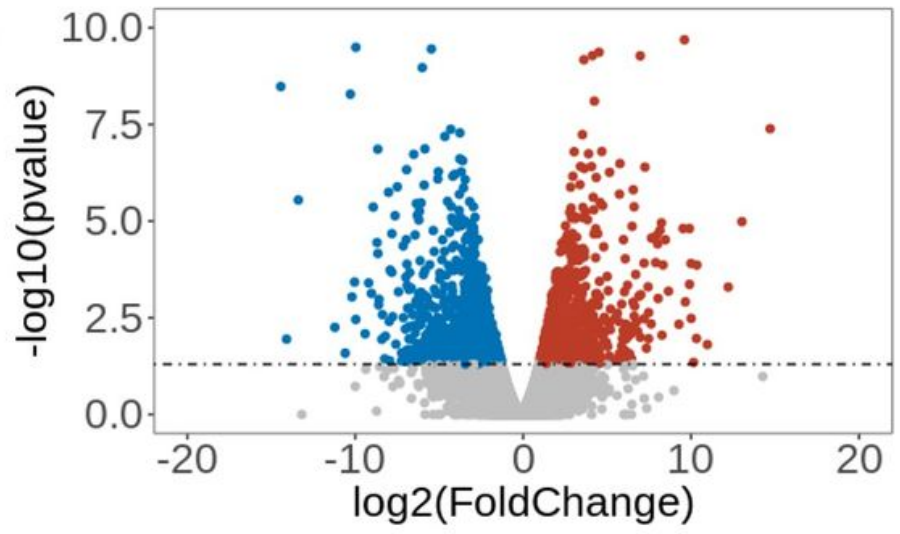

(C)

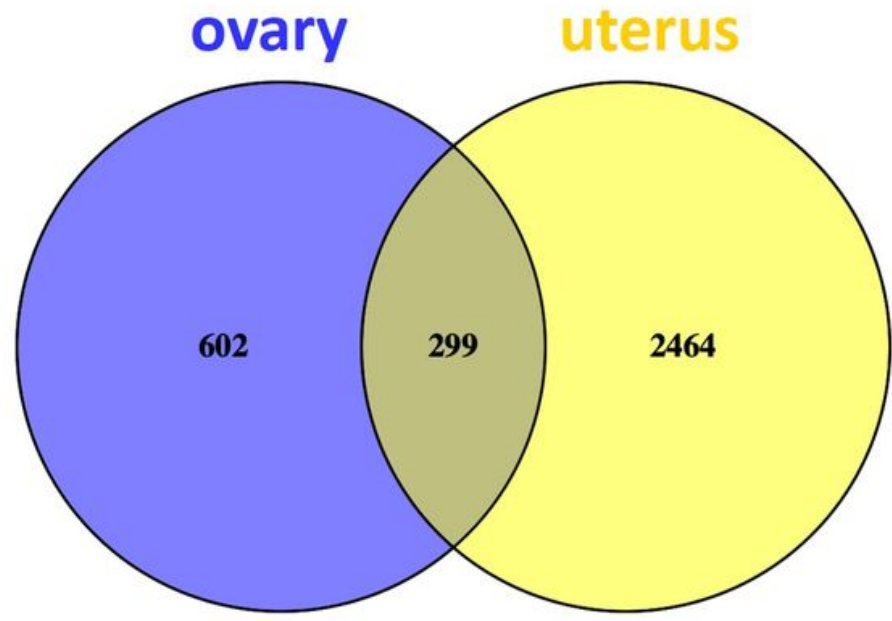

Figure 2

Volcano plot and Venn diagram of DEGs. (A) and (B) present the volcano plot of DEGs in ovary and uterus, respectively. (C) Venn diagram of DEGs between ovary and uterus. 

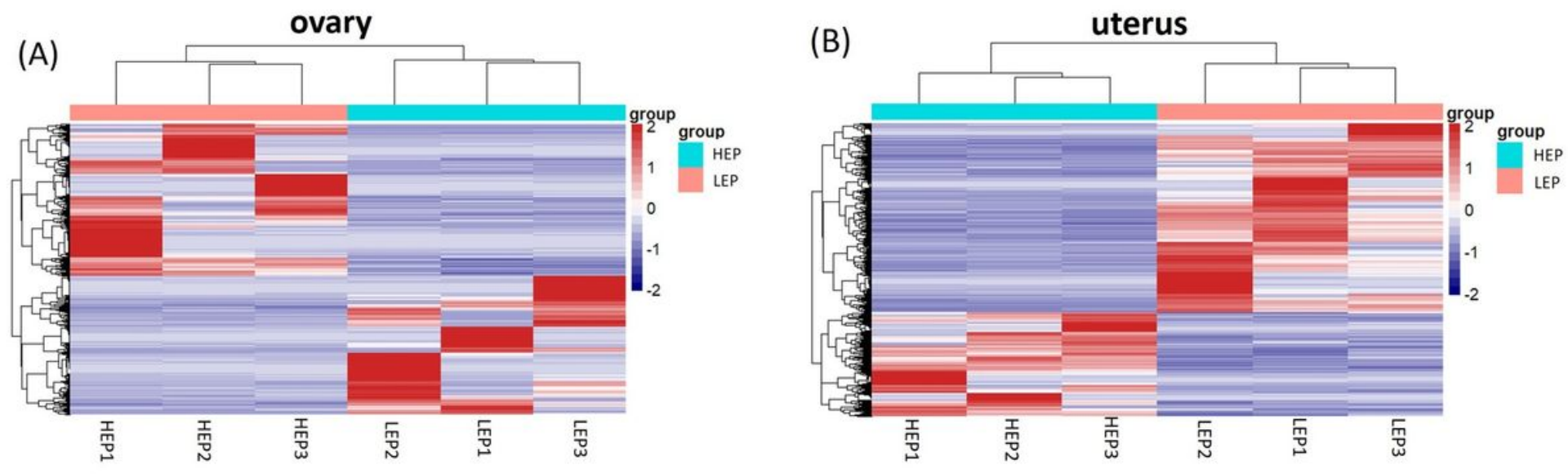

Figure 3

Hierarchical clustering analysis of the significantly DEGs between HEP and LEP in ovary and uterus. (A) 602 DEGs in the ovary; (B) 2464 DEGs in the uterus. Red present up-regulated genes, green present down-regulated genes.

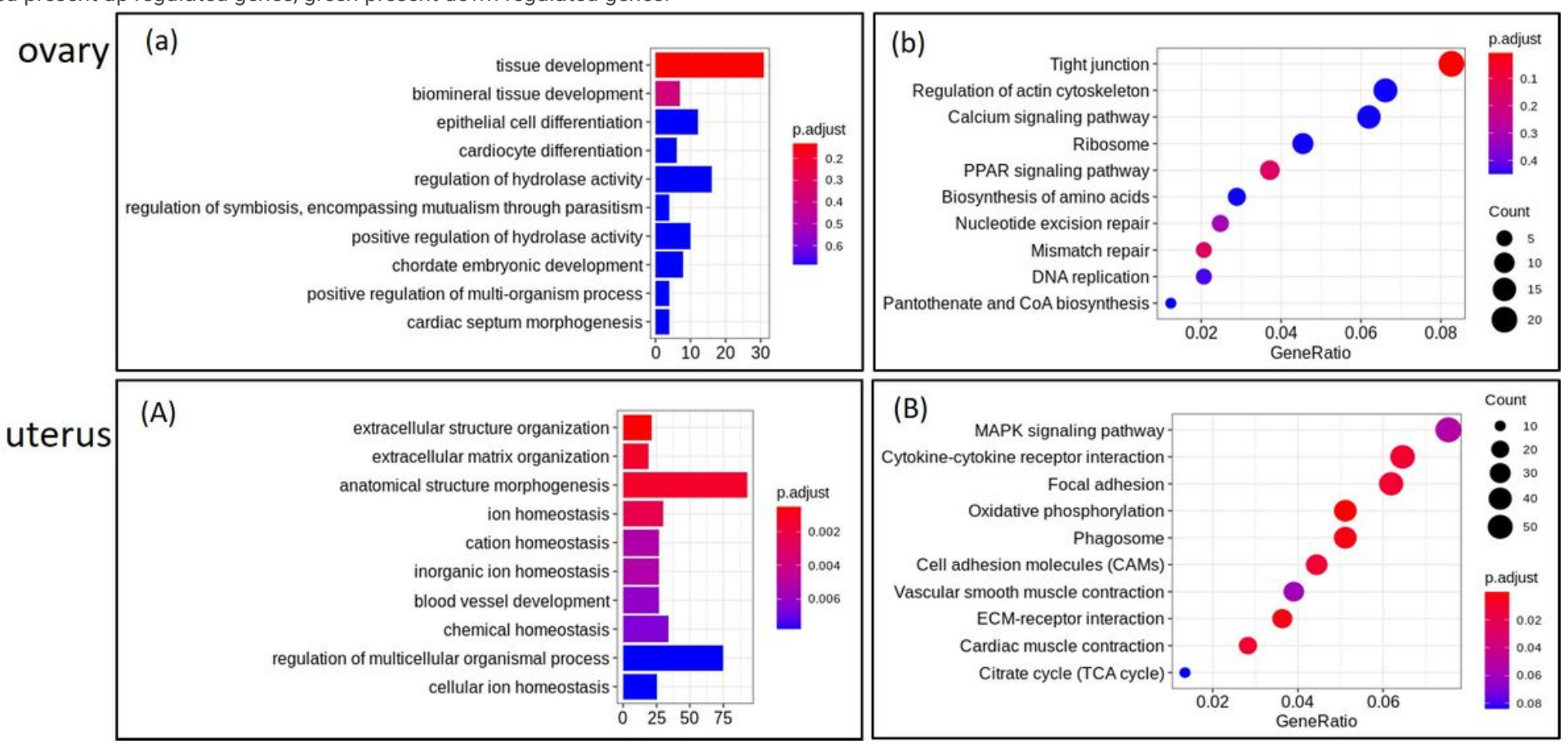

\section{Figure 4}

GO and KEGG enrichment analysis of DEGs. (a) and (b) present GO enrichment and KEGG analysis of DEGs in the ovary, respectively. (A) and (B) present GO enrichment and KEGG analysis of DEGs in the uterus, respectively. 

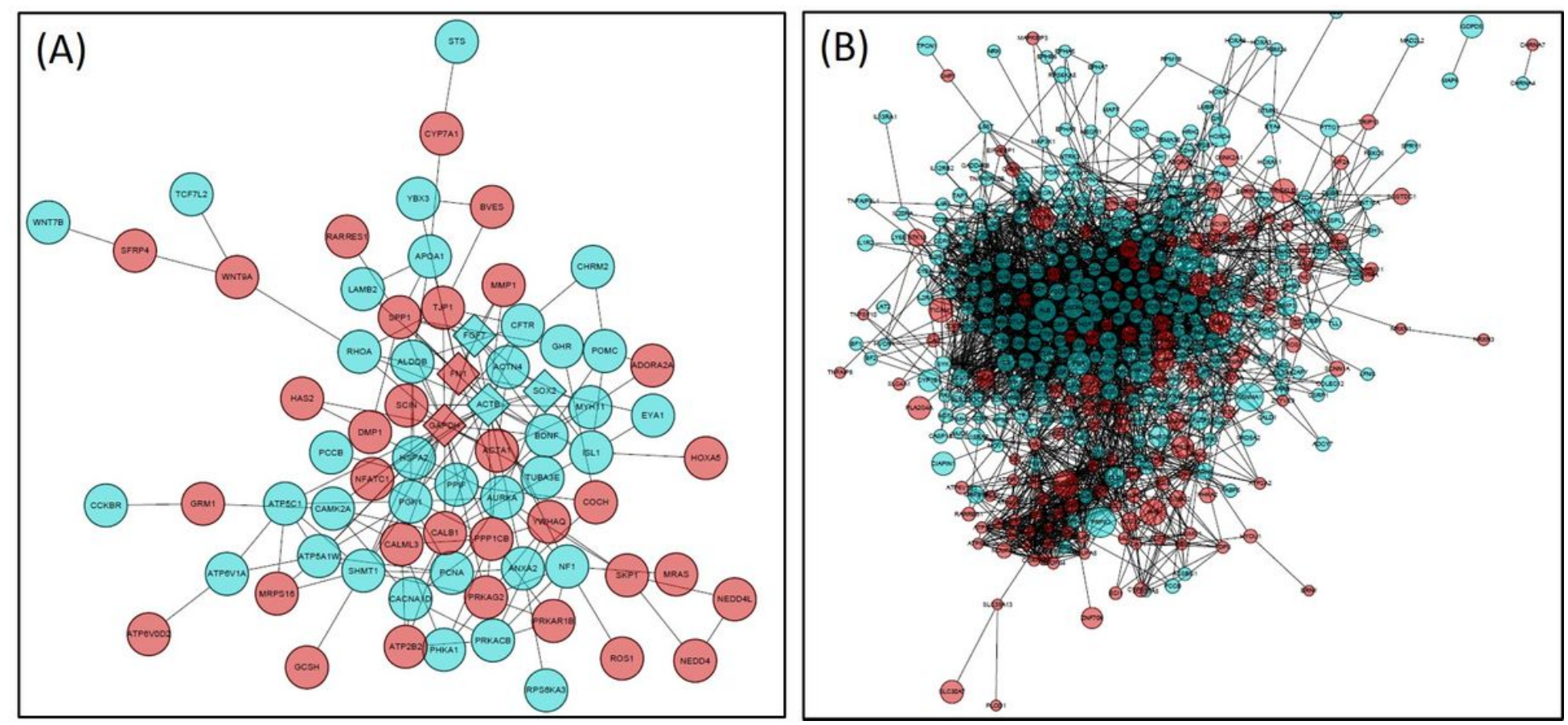

\section{Figure 5}

Protein-protein interaction (PPI) network for DEGs in ovary and uterus. A total of 77 and 428 nodes, 175 and 2962 interaction associations in ovary and uterus, respectively. Red and blue nodes present up-regulated genes and down-regulated genes, respectively. Diamond is the nodes with the highest degree scores, while the size of the node is the expression levels of genes.
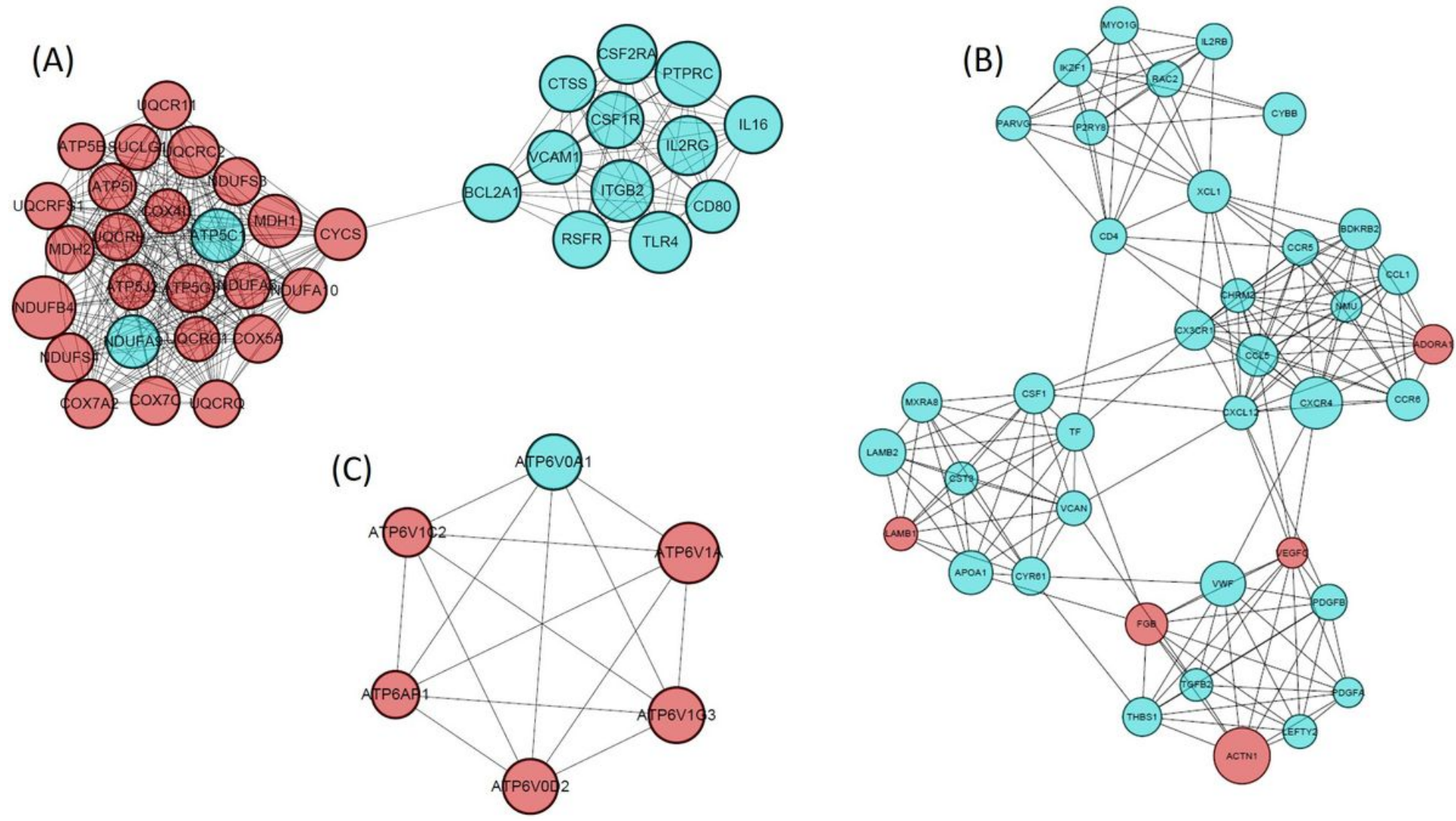

\section{Figure 6}

Three protein-protein interaction (PPI) hub network modules in the uterus. The three significant modules, (A) module 1 (MCODE score =18.579), (B) module 2 (MCODE score $=10)$, and $(C)$ module 3 (MCODE score $=6$ ), were constructed using PPI network of DEGs. Red and blue nodes present up-regulated genes and down-regulated genes, respectively. The size of the node is the expression levels of genes. 

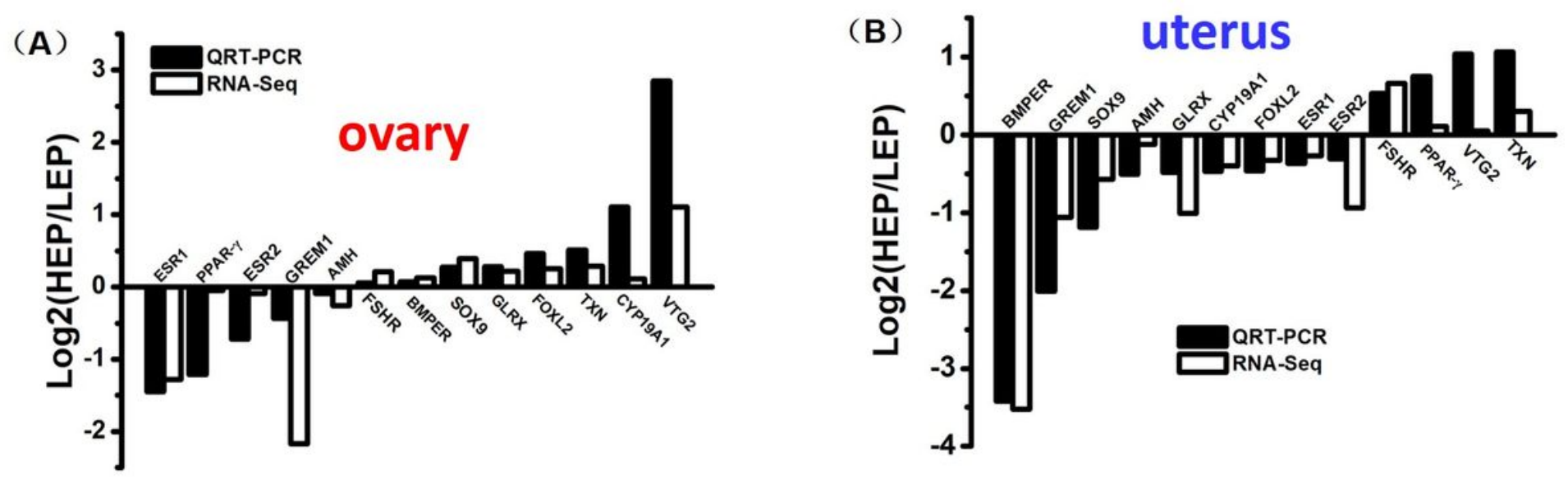

Figure 7

RNA-Seq validation using QRT-PCR. 13 DEGs were selected randomly to test the accuracy of RNA sequencing. (A) validation in the ovary. (B) validation in the uterus. $\mathrm{N}=6$.

\section{Supplementary Files}

This is a list of supplementary files associated with this preprint. Click to download.

- NC3RsARRIVEGuidelinesChecklistfillable.pdf

- Tables1.docx 